\section{New influenza virus in horses}

SIR - In March 1989, an outbreak of severe respiratory disease occurred in horses in the Jilin and Heilongjiang provinces of northeastern China (Y. Guo et al. Chinese J. exp. clin. Viroi. 3, 318-322; 1990). Morbidity was 81 per cent and mortality as high as 20 per cent in some herds. In Zhilia county within Jilin province, 13,151 horses were infected and the mortality reached 35 per cent. Peaking in late March and April, the outbreak had abated by June 1989. Disease onset was acute, with coughing, bronchitis, pneumonia and fever of more than $40^{\circ} \mathrm{C}$. Animals were fatigued with inflammation of the nasal mucosa and copious mucous discharge from the eyes and nose. Most cases lasted for 5-6 days, but some animals showed symptoms for 2 weeks. Death was associated with pneumonia and enteritis. Similar signs of disease were noted in mules but not in donkeys. None of the people who worked with the diseased horses had signs of respiratory disease. A second outbreak occurred in April 1990 in Heilongjiang province, resulting in 41 per cent morbidity but no deaths.

An influenza virus of the H3N8 subtype was isolated from the infected animals and shown to be antigenically and molecularly distinct from the equine 2 (H3N8) and human $\mathrm{H} 3 \mathrm{~N} 2$ viruses currently circulating in the world. Sequence analysis of the entire haemagglutinin, nucleoprotein, matrix and nonstructural genes, together with partial sequencing of the three polymerase and the neuraminidase genes, established that five of the eight gene segments of this new virus, A/Equine/Jilin/1/89 (H3N8), are most closely related to avian influenza viruses of

\section{Striding on water}

SIR - Daedalus has suggested a 'Seasurfer sea-going platform' based on his recent invention, graphite-fluoride flock ${ }^{1}$. Water striders (Hemiptera: Gerridae) have also exploited the hydrophobic properties of closepacked fibres to walk on water ${ }^{2}$. Daedalus should consider the advantages inherent in constructing a platform based on water striders; the ability to move into the wind or up a wave slope to mention two. Furthermore, the ability of water striders to communicate by vibrating their forelegs on the water-surface membrane ${ }^{3}$ means that a Seasurfer constructed of water striders would come with its own built-in mobile phone.

Stephen J. CLARK

Field Research Center for Ecology and Ethology,

The Rockefeller University,

Tyrrel Road, Millbrook,

New York 12545, USA

1. Jones, D. Nature 350, 196 (1991).

2. Cheng, L. Nature 242, 132-133 (1973)

3. Wilcox, R. S. Science 206, 1325-1327 (1979).
Eurasian origin. The polymerase genes (PB1 and PB2) are genetically conserved between all influenza virus isolates and their origin could not be determined unequivocally. The partial sequence of the neuraminidase gene of the new $\mathrm{H} 3 \mathrm{~N} 8$ isolate was distinguishable from current equine H3N8 viruses, but limited data preclude establishment of its origin. (Y. Guo et al., unpublished data.) Phylogenetic analysis indicated that four of the eight genes were recently derived from avian sources. The nucleoprotein gene was closely related to that found in the newly described $\mathrm{H} 14$ subtype isolated from ducks in the Soviet Union. On initial inoculation, the A/Equine/Jilin/1/89(H3N8) influenza virus failed to replicate in ducks, but did replicate and cause disease in mice; on subsequent passaging it caused 100 per cent mortality. It caused severe influenza symp-

The evidence suggests that this new equine influenza virus in northeast China is the latest mammalian influenza virus to emerge from the avian gene pool and that it has possibly spread to horses without gene reassortment. This direct transmission suggests that avian influenza viruses with this combination of surface proteins $(\mathrm{H} 3 \mathrm{~N} 8)$ have a selective advantage over other subtypes for replication in horses. Seroarchaeological studies indicate that an H3N8 virus appeared in 1890 in the human population, coincident with historical records of an influenza pandemic in that year. Phylogenetic analysis of nucleoprotein genes suggests that the equine 2 virus genome originated in the late nineteenth century. Thus, one is tempted to speculate that the H3N8 human and equine viruses had similar origins.

The last known instance of an avian influenza virus transferring its entire genome to mammals and establishing a successful epidemic was in pigs in Europe (C. Scholtissek, H. Burger, P. A. Bachmann \& C. Hannoun Virology 129, 521-523; 1983). This avian-like swine influenza virus replaced classical swine influenza virus in pigs in Europe in the 1980s and has since become endemic in that swine population. There have probably been other successful transfers of intact avian influenza viruses to mammals. Available evidence suggests that the virus responsible for Spanish influenza in 1918-19 derived all its genes from an avian reservoir and entered the human population before 1918. The appearance of the new equine influenza virus in China emphasizes the possibility of whole avian influenza viruses entering mammalian hosts and serves as a warning that a new outbreak of human influenza could occur by this mechanism.

Will the outbreak in horses be limited to China, or could it become global? The answer will depend on the movement of horses from this region. Because there is trade across the Sino-Soviet border, the most toms in ferrets. likely route for spread is through the Mongolian horse population to central Russia. People who heep horses should be aware of the presence of this virus and maintain quarantine practices to reduce its spread. One important consideration is that, although the virus originated recently from birds, it seems to have lost its ability to replicate in ducks, reducing the likelihood of spread by aquatic birds during migratory flights.

Department of Virology and

Molecular Biology,

St Jude Children's Research Hospital,

332 North Lauderdale,

PO Box 318, Memphis,

Tennessee 38101, USA

National Influenza Centre,

GUO YUANJI

Department of Influenza,

Institute of Virology,

Chinese Academy of Preventive

Medicine,

Beijing 100052,

China

\section{Relationship of FKBP to PKCl-2}

SIR - Goebl et al. in Cell ${ }^{1}$ and Tropschug and Hofmann in Scientific Correspondence ${ }^{2}$ have reported the near-identity of the amino-acid sequence of the human FK506and rapamycin-binding protein (FKBP) ${ }^{3}$ with a bovine protein, termed PKCI-2 (ref. 4 ), which is purported to inhibit the enzymatic activity of protein kinase C (PKC). These authors suggested that PKC is the target of the immunophilin-drug complex ${ }^{2}$. Indeed, the sequence reported for PKCI-2 is identical to the amino-acid sequence of bovine FKBP purified from thymus ${ }^{5}$.

We have investigated the effect of FKBP on phosphorylation mediated by $\mathrm{PKC}$ and find that FKBP and its ligands FK506 and rapamycin, either acting alone or bound to FKBP, do not inhibit the kinase activity of isolated PKC or PKC-mediated events in cells ${ }^{6}$. Moreover, similar results have been obtained with cyclophilin and cyclosporin A (CsA). Thus, in the light of these biochemical and cellular studies, we surmise that the target protein(s) of immunophilin-drug complexes (FKBP-FK506, FKBP-rapamycin and cyclophilin-CsA) is not PKC.

Mark W. Albers JUN LIU StUART L. Schreiber

Department of Chemistry,

Harvard University,

Cambridge,

Massachusetts 02138, USA

1. Goebl, M. G. et al. Cell 64, 1051-1052 (1991).

2. Tropschug, M. \& Hofmann, R. Nature 351, 195 (1991).

3. Standaert, R. F., Galat, A., Verdine, G. L. \& Schreiber, S. L Nature 346, 671-674 (1990).

4. Mozier, N. M. et al. Eur. J. Biochem. 194, 19-23 (1990)

5. Lane, W. S., Galat, A., Harding, M. W. \& Schreiber, S. L. J. prot Chem. 10, 151-160 (1991).

6. Albers, M.W. etal. BioMed. Chem. Lett.1, 205-210(1991). 